Paediatr. Paedolog. 2022 · 57:1

https://doi.org/10.1007/s00608-021-00955-w

Angenommen: 7. Dezember 2021

(C) The Author(s), under exclusive licence to Springer-Verlag GmbH Austria, ein Teil von Springer Nature 2021

\section{Josef Riedler}

Kardinal Schwarzenberg Klinikum GmbH, Schwarzach im Pongau, Österreich
Wikipedia definiert die Ökonomisierung als die Ausbreitung des Markts bzw. seiner Ordnungsprinzipien und Prioritäten auf Bereiche, in denen ökonomische Überlegungen in der Vergangenheit eine eher untergeordnete Rolle spielten. So werden zunehmend immer mehr Güter und Praktiken, die einst außerhalb der Marktsphäre lokalisiert waren, in „Produkte" umgewandelt, die über einen Preis auf einem Markt gehandelt werden können. Dies trifft im Allgemeinen auf die soziale Arbeit und im Besonderen auf die Medizin mit gravierenden Konsequenzen zu.

In der Medizin führt Ökonomisierung in erster Linie zu einer Zunahme der Geschwindigkeit, mit der Patienten behandelt werden sollen, $\mathrm{zu}$ einer Steigerung der Patientenzahlen und $\mathrm{zu}$ einer Reduktion von Spitalsbetten und Vorhaltekapazitäten. Der Faktor Zeit ist das größte Ökonomisierungspotenzial. Wieviel Vertraulichkeit und Individualität kann es geben, wenn an einem Vormittag in einer Allgemeinmedizinerordination 100-150 Patienten durchgeschleust werden? Ist Ökonomisierung mit Individualität überhaupt kompatibel?

Auch medizinische Leitlinien können Bestandteil eines Ökonomisierungsprozesses sein. Leitlinien sind systematisch entwickelte, evidenzbasierte medizinische Handlungsempfehlungen, die die Betreuung von Patienten aufgrund objektiver Daten standardisieren. Für das ärztliche Handeln sind sie grundsätzlich sehr sinnvoll, da ihre Verwendung nachvollziehbare Betreuungsstandards sichert und eine rein subjektive ärztliche Behandlungsbeliebigkeit verhindert. Digital sehr schnell verfügbare Leitlinien dürfen jedoch nicht zu einer Kommerzia- lisierung der Patientenbetreuung führen. Um einen Patienten personalisiert und präzise behandeln zu können, muss der Arzt die Freiheit haben, in begründeten Situationen von der Leitlinienempfehlung abzuweichen. Personalisiert bedeutet nämlich nicht, ausschließlich eine Clustermedizin nach für Gruppen zutreffenden objektiven Biomarkern $\mathrm{zu}$ betreiben, sondern auch das soziale Umfeld, die kognitiven Fähigkeiten und den kulturellen Hintergrund des einzelnen Patienten zu berücksichtigen.

Wird der Patient als ,industrialisiertes Produkt" gesehen, schafft die Ökonomisierung die ärztliche Kunst ab, um in den Worten von Giovanni Maio, einem führenden deutschen Medizinethiker zu sprechen. Zur ärztlichen Kunst zählen auch zuhören, Zeit schenken, Verständnis zeigen und vor allem Vertrauen aufbauen.

Diese Aspekte einer Patient-Arzt-Interaktion sind nicht nur, aber ganz besonders, in der psychosomatischen Medizin von eminenter Bedeutung. Und es verwundert nicht, dass ausgerechnet die verschiedenen Formen der psychosomatischen Krankheiten weltweit stark im Zunehmen sind, denn sie sind aufwendig in der Behandlung und benötigen viel Zeit, die in der ökonomisierten Medizin nicht vergütet wird. Ein Hin- und Herschieben von Patienten zwischen verschiedenen medizinischen Berufsgruppen ist die Folge mit allen negativen Konsequenzen wie Patientenunzufriedenheit, „doctor shopping" und Kostensteigerung.

Die Kinder- und Jugendmedizin leidet besonders unter der Ökonomisierung, da die Behandlung von kranken Kindern und die Betreuung der besorgten Begleitpersonen (meist Mutter oder Vater) sehr individuell und ausgesprochen zeitinten- siv sind. Der Informations- und Erklärungsbedarf von Eltern ist im digitalen Zeitalter rasant angestiegen.

Dass Ärzte bei ihrer Arbeit vorhandene Ressourcen schonen und ökonomisch denken (aber nicht zwangsläufig kommerziell handeln), ist ein unverzichtbarer Teil ihres Berufsethos und widerspricht nicht einem medizinisch-ethischen Handeln. Ihre Arbeit jedoch einer radikalen Ökonomisierung zu unterjochen, ist unethisch und nicht vertretbar. Unter so ein Joch fällt auch die Durchführung finanziell lukrativer Untersuchungen und Behandlungen, die medizinisch nicht unbedingt indiziert sind, aber auch das ungerechtfertigte Weglassen teurer Maßnahmen, um Kosten zu sparen.

Die Medizin muss menschlich und zuwendend sein sowie frei von rigorosen ökonomischen Zwängen, um ihrem Auftrag nach ganzheitlicher Hilfe und im besten Fall Heilung von Patienten (und nicht Kunden) gerecht zu werden.

\section{Korrespondenzadresse

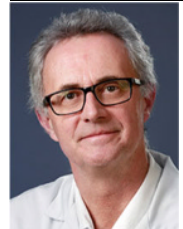 \\ Prim. Univ. Prof. Dr. Josef Riedler, FERS Kardinal Schwarzenberg Klinikum GmbH Kardinal Schwarzenberg- platz 1, 5620 Schwarzach im Pongau, Österreich josef.riedler@ks-klinikum.at}

Interessenkonflikt. J. Riedler gibt an, dass kein Interessenkonflikt besteht.

Hinweis des Verlags. Der Verlag bleibt in Hinblick auf geografische Zuordnungen und Gebietsbezeichnungen in veröffentlichten Karten und Institutsadressen neutral. 